\title{
VISUALIZATION OF MYOCARDIAL INFARCTION AND SUBSEQUENT CORONARY REPERFUSION WITH MRI USING A DOG MODEL
}

\author{
Alex M. Aisen,* Andrew J. Buda, $\nmid$ Rainer J. Zotz $\dagger$ and Kenneth A. Buckwalter* \\ Department of Radiology and the Cardiology Division, †Department of Internal Medicine, \\ University of Michigan Hospitals, Ann Arbor, Michigan 48109, USA
}

\begin{abstract}
Twelve anesthetized mongrel dogs underwent left thoracotomy with placement of a removable ligature around the left circumflex coronary artery. Following a 3 to 6 hour delay, ECG-gated spin-echo MRI was performed. The ligature was then removed reperfusing the heart, and after a 10-15 min period, MRI repeated. Finally, postsacrifice images were obtained, and the hearts chemically stained for infarct evaluation. The MR images were subjectively and quantitatively evaluated for visibility of the endocardial border and of the injured myocardium, and for changes after reperfusion. The injured tissue was variably visible in vivo, the major limitation a result of motion blurring and artifact. The abnormal tissue was easily visible on MRI in 11 animals, and not clearly visible in one. The endocardial border was easily seen in 10 animals. The variation of calculated relaxation times was high for both normal and ischemic/infarcted myocardium in the beating hearts (normal: $T 1=566 \pm 288$, $T 2=38 \pm 6$; injured myocardium: $T 1=637 \pm 250, T 2=41 \pm 12$ ) in contrast, relatively stationary skeletal muscle measured in the same images had narrower ranges $(T 1=532 \pm 199, T 2=28 \pm 2)$. Changes with reperfusion were seen, but not reliably. The infarcted or ischemic zones were easily visible on post-sacrifice images in all animals imaged. Post-sacrifice relaxation times were $T 1=564 \pm 69 \mathrm{msec}, T 2=39 \pm 3$ msec for normal heart muscle, and $725 \pm 114, T 2=47 \pm 5$ for ischemic/infarcted tissue. We conclude that acute myocardial infarction can usually be detected by MRI, given a prior knowledge of its location. However, the technique is at present likely to be of only limited value clinically in the prospective diagnosis of acute myocardial infarction, though this may improve as technology advances. Finally, signal changes following reperfusion may be visible in some cases, but not reliably so.
\end{abstract}

\section{INTRODUCTION}

The ability of proton MR imaging to demonstrate acute myocardial infarction as areas of increased signal on spin-echo images has been demonstrated both in animal models ${ }^{5,12,14,18}$ and in man..$^{3,4,9,11}$ The current investigation was undertaken to provide additional information on the reliability of MR in detecting acute myocardial injury, and thus to provide an indication of the potential role of current MR equipment in a clinical setting.

\section{MATERIALS AND METHODS}

Twelve mongrel dogs were anesthetized with intravenous pentobarbital $(30 \mathrm{mg} / \mathrm{kg})$, intubated, and ven- tilated artificially with room air. A thoracotomy was performed, and a left atrial catheter placed for subsequent injection of technetium-99m labeled microspheres. A proximal segment of the left circumflex coronary artery was dissected free, and a removable ligature was then tied around the vessel, occluding it. Following occlusion, approximately 400,000 to 600,000 technetium-99m labeled 20 micron microspheres were injected.

A mean period of 4 hours (range, 2.5-6) after occlusion, the animal underwent ECG-gated MR using a Diasonics MT/S whole body imager, with a $0.35 \mathrm{~T}$ superconducting magnet. Dual spin-echo, multislice acquisition in the transverse plane was employed, with echo-delay (TE) times of 28 and $56 \mathrm{msec}$. Cardiac gat-
RECEIVED 2/5/87; ACCEPTED 5/22/87.

Acknowledgments - This work was supported, in part, by a Grant-in-Aid from the American Heart Association of Michigan and by Grant HL29716 from the National, Heart, Lung, and Blood Institute, National Institutes of Health, Bethesda, MD. Portions of this work were presented at the American Federation for Clinical Research, Washington, DC, May 3-6, 1986.
Address correspondence and reprint requests to Alex $\mathrm{M}$. Aisen, M.D., Department of Radiology/MRI - Box 0030, University of Michigan Hospitals, 1500 E. Medical Center Drive, Ann Arbor, MI 48109.

$\ddagger$ Present address: II Medizinische Klinik, ABT. Kardiologie, Langendeck STR, 6500 Mainz, West Germany. 
ing was performed using two different pulse-repetition (TR) intervals for each study; for animals with a slow heart rate, gating to every beat and every other beat was used; for those with a faster rate, gating was typically to every second and every third beat. The average TR's were $0.81 \pm 0.18 \mathrm{sec}$ for the shorter gating interval, and $1.31 \pm 0.38$ for the longer.

Following the initial MR study, the animal was withdrawn from the imaging magnet, and the coronary ligature removed. The animal was reinserted into the scanner; care was taken to insure that the same position was used. After a period of from 10 to 15 minutes, a second set of images were acquired, again with two different gating intervals.

Three of the animals then received injections of gadolinium-DTPA for a study unrelated to this investigation. All animals were sacrificed by potassium chloride injection, and post-sacrifice scans were obtained in all animals except one of the gadolinium injected dogs using TR intervals of 0.5 and 1.0 seconds. In 3 animals, these images were obtained on the excised heart; in the remainder, the heart was imaged in situ.

Following sacrifice, all hearts were excised. The infarcted myocardium was identified and sized using triphenyltetrazolium (TTC) staining, and the volume of myocardium hypoperfused during occlusion was measured using autoradiography, as previously described. ${ }^{2}$

For all sets of MR images, the slice best displaying the cardiac anatomy was selected. The time interval following the QRS trigger for this slice varied from case to case; generally, it proved to be the slice acquired 100 or 200 milliseconds after the QRS complex. For almost all animals, the same anatomic level was used for all measurements; in a small number of cases, it was necessary to use contiguous slices which differed by $1 \mathrm{~cm}$. The 4 pre-reperfusion images (obtained with 2 gating intervals and $2 \mathrm{TE}$ times), 4 post-reperfusion images, and 4 post-sacrifice images (excluding the gadolinium injected animals) were evaluated visually and quantitatively. Visually, the following determinations were independently made by 2 radiologists (AMA and $\mathrm{KAB}$ ), and a consensus reached for: visibility of the endocardial border on the 4 pre-reperfusion images; visibility of injured myocardium as an area of increased signal in the region of posterior left ventricle perfused by the circumflex artery; presence of a change in contrast between normal and abnormal myocardium following reperfusion; and visibility of injured myocardium on the post-sacrifice images.

Regions of interest were electronically drawn under visual guidance through zones of normal myocardium and the areas with increased signal, and average pixel intensities measured. From these data, calculations of
$T 1$ and $T 2$ relaxation times were made in the pre-reperfusion, post-reperfusion, and post-sacrifice images (the latter excluding the animals given gadoliniumDTPA), and ratios of signal intensity in the normal and abnormal myocardium determined for the firstecho image data (again excluding post-sacrifice data on the injected animals). Regions of interest were also drawn in areas of skeletal muscle, and relaxation times calculated. Standard software furnished with our scanner was used for the calculations, as described by other investigators. ${ }^{18}$ Results are expressed as mean \pm S.D.

\section{RESULTS}

\section{Postmortem}

The radio-labeled microsphere assessment of the hypoperfused region was technically unsuccessful in one animal; in the remaining 11 animals, the mean ratio of hypoperfused myocardium (risk region) to total left ventricular myocardium was $34 \pm 7 \%$. TTC staining was also technically unsuccessful in one animal; of the remaining 11 dogs, infarcts were observed in 10; injury in one animal apparently did not progress to infarction. The mean infarct size for the 10 dogs was $22 \pm 12 \%$ of the myocardium.

\section{Visual analysis}

In general, in vivo second echo images were of poorer quality than first echo data, because of their lower signal-to-noise, and the presence of increased motion artifact. It was, however, preferable to examine both echo images concurrently; this generally permitted identification of the area of injury more easily than on either first or second echo images interpreted alone (see Figs. 1a and 1b).

On the pre-reperfusion images it was almost always possible to identify the injured myocardium as an area of increased signal intensity in the expected location. Visibility was generally better on the longer TR images; for the shorter TR first and second echo images read together, the injury could be easily identified in $7 / 12$ $\operatorname{dogs}(5 / 12$ for first echo images alone, and $3 / 12$ for second echo images alone), for the longer TR, in $11 / 12$ animals $(11 / 12$ for first echo images alone, $8 / 12$ for second echo images alone). However, areas of increased signal in areas of myocardium not corresponding to the expected location of tissue injury was noted in $7 / 12$ animals, almost certainly a result of motion artifact. Thus, we must note that without prior knowledge of infarct location, reliable diagnosis would generally not have been possible.

The endocardial border was easily discerned in 10 dogs on both the short and long TR images, and not well seen in 2 . 

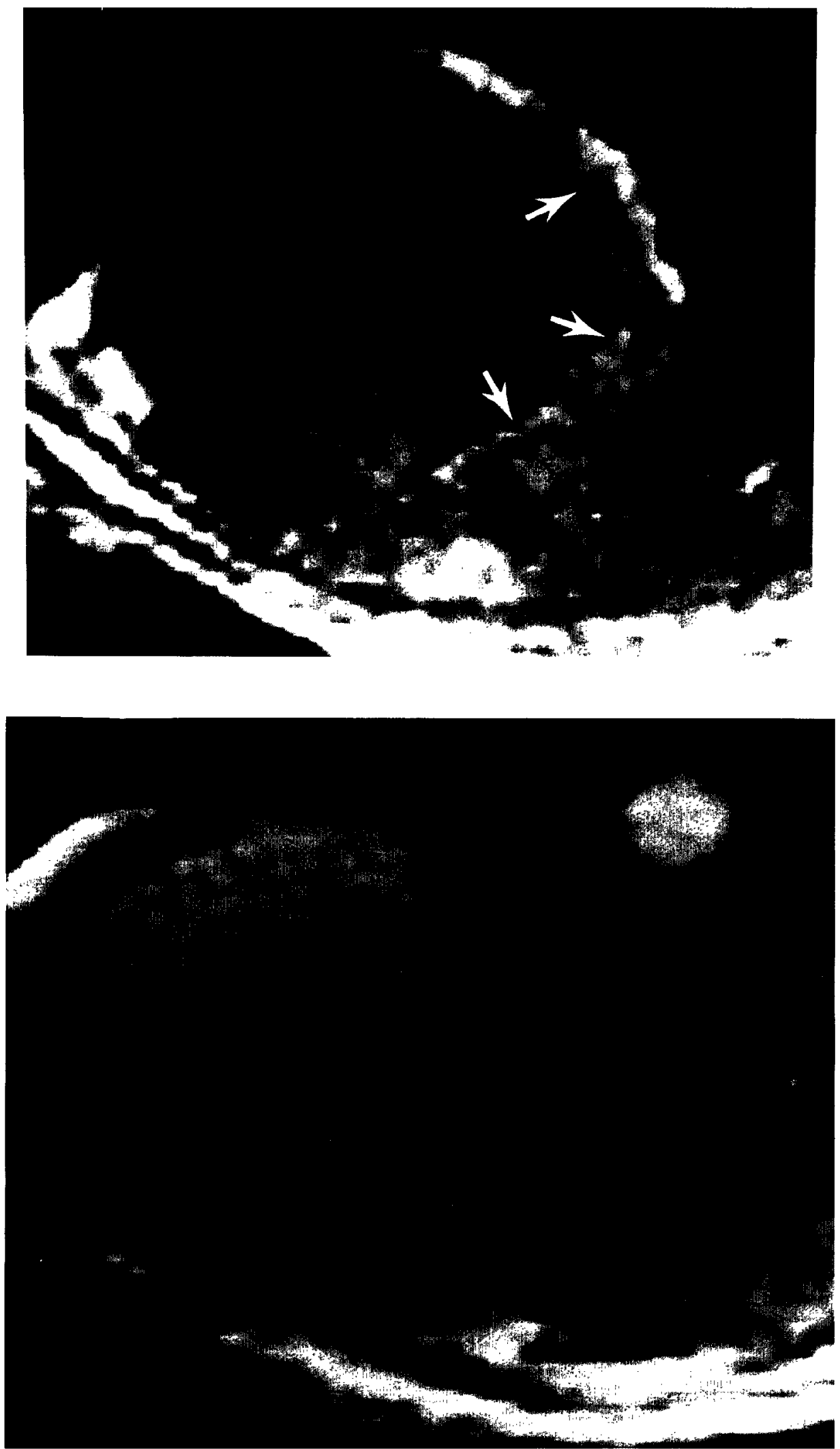

Fig. 1. (A) Short TR $(0.6 \mathrm{sec})$, first echo image demonstrating the myocardial injury as an area of increased signal (arrows). (B) $\mathrm{TR}=1.0, \mathrm{TE}=56 \mathrm{msec}$ image obtained after sacrifice confirming the presence of a high signal zone. 


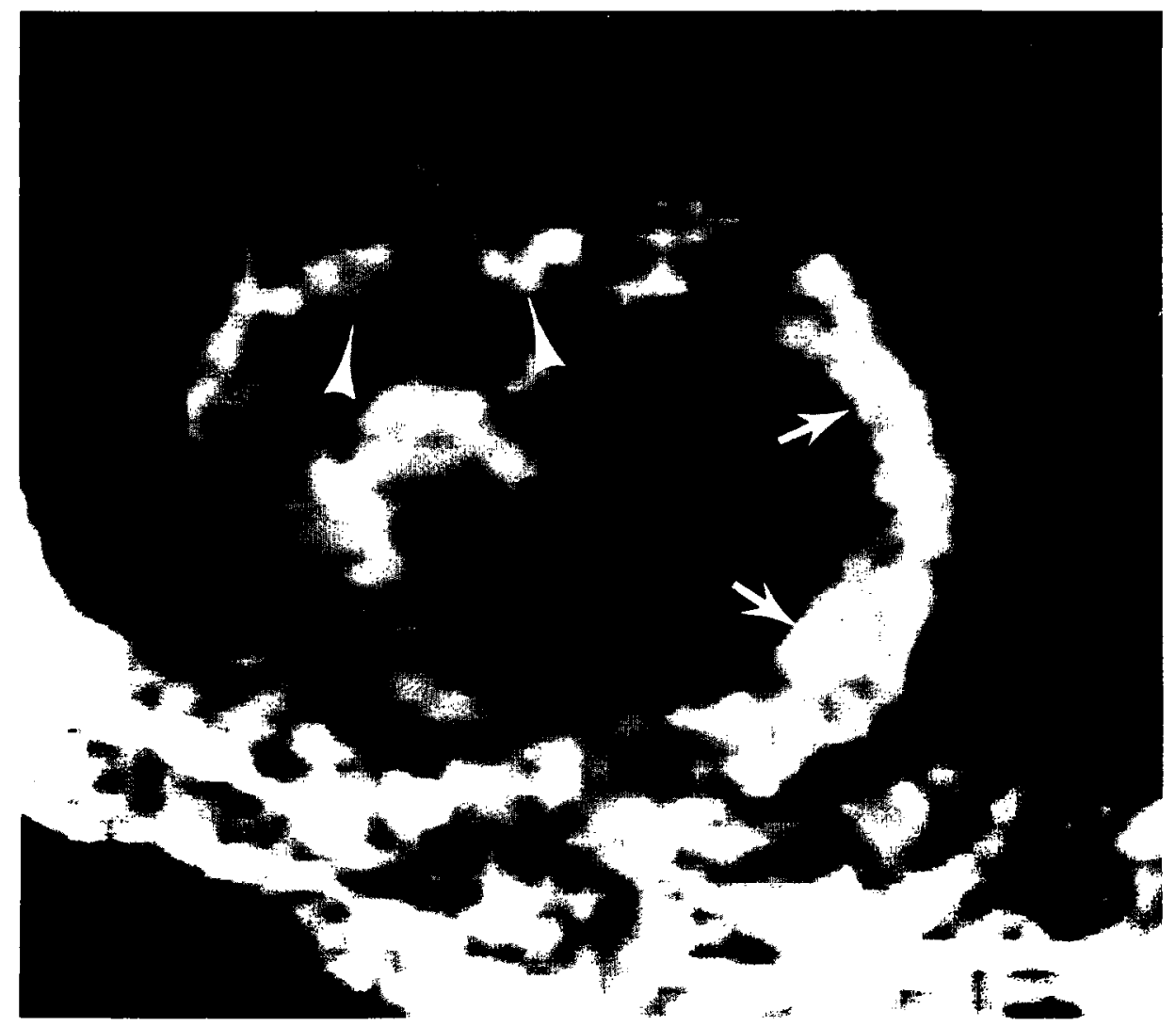

Fig. 2. Long TR $(1.67 \mathrm{sec})$, first echo image $(28 \mathrm{msec})$ with areas of increased signal due to both injury (arrows) and apparent motion artifact (arrowheads).

It was not felt possible to visually determine whether or not a change in visibility of the injured myocardium occurred with immediate reperfusion.

The injured myocardium was clearly seen in all 9 animals (the 3 animals given Gd-DTPA are excluded) following sacrifice on the TR $=1.0$ second images (first echo TE $=28 \mathrm{msec}$ and second echo $\mathrm{TE}=56$ msec data alike), and in 3 of the animals on the TR = 0.5 second, $\mathrm{TE}=56 \mathrm{msec}$ data (it could be identified in only one dog on the TR $=0.5 \mathrm{sec}, \mathrm{TE}=28 \mathrm{msec}$ scans). Visibility was much improved over the in vivo images (Fig. 2).

\section{Quantitative}

The ratio of signal intensity in the injury zone to normal myocardium was greater than unity in all but one first-echo image. For the pre-reperfusion images, the mean intensity ratio was $1.16 \pm 0.23$ for the shorter TR images, and $1.40 \pm 0.25$ for those obtained with the longer TR. For the post-reperfusion scans, the ratios were $1.31 \pm 0.23$ and $1.49 \pm 0.45$, for the short and long TR data, respectively. A trend toward increasing lesion contrast following reperfusion is present, but it did not reach statistical significance. Additionally, a trend was noted towards increasing lesion contrast on the more heavily $T 2$-weighted long TR images compared to the short TR-images; however, this reached statistical significance $(p<0.05)$ for the prereperfusion data only.

The calculated relaxation time data is summarized in Table 1. As can be seen, the variation in these measurements was great, severely limiting their value. The variation in $T 2$ times was less than for the $T 1$ values; this is usually the case with image data, and probably results from the fact that $T 2$ calculations are based on data acquired in a single dual-echo pulse sequence, whereas $T 1$ calculations require two separate sequences, with different TR intervals. Variation in the measurements for skeletal muscle, which of course does not experience cardiac motion, was substantially less than for myocardium. Finally, the variation found following sacrifice was generally substantially less than in the live animal.

The relaxation time data did not show significant 
Table 1. Relaxation times

\begin{tabular}{llc}
\hline & \multicolumn{1}{c}{$T 1^{*}$} & $T 2$ \\
\hline Normal Myocardium, pre-reperfusion & $566 \pm 288(9)$ & $38 \pm 6(12)$ \\
Normal Myocardium, post-reperfusion & $521 \pm 212(10)$ & $42 \pm 11(12)$ \\
Normal Myocardium, post-sacrifice & $564 \pm 69(9)$ & $39 \pm 3(9)$ \\
Injured Myocardium, pre-reperfusion & $637 \pm 250(11)$ & $41 \pm 12(12)$ \\
Injured Myocardium, post-reperfusion & $647 \pm 213(11)$ & $45 \pm 11(12)$ \\
Injured Myocardium, post-sacrifice & $725 \pm 114(9)$ & $47 \pm(9)$ \\
Skeletal Muscle, pre-reperfusion & $532 \pm 199(11)$ & $28 \pm 2(12)$ \\
Skeletal Muscle, post-reperfusion & $544 \pm 199(11)$ & $28 \pm 1(12)$ \\
Skeletal Muscle, post-sacrifice & $593 \pm 186(6)$ & $29 \pm 1(6)$ \\
\hline
\end{tabular}

Mean \pm standard deviation, in milliseconds. Number in parentheses indicates number of usable data points.

* In several cases, the data did not permit calculation of the $T 1$ relaxation time (the heart rate was not recorded for one animal; in 3 other measurements, the measured long-TR intensity was found to be less than the short-TR intensity).

differences in relaxation times between normal and ischemic myocardium in the living animal, though a trend towards increased relaxation times is evident. In the measurements made after sacrifice, the differences are statistically significant. No significant differences between the relaxation times of injured myocardium before and after reperfusion were observed.

\section{DISCUSSION}

We have assessed the efficacy of different pulse sequences for spin-echo MRI in visualizing acute myocardial infarction in a controlled dog model. Previous reports have shown that infarcts are seen as zones of increased signal intensity..$^{5,11,12,14,18}$ However, several recent patients have indicated controversy over the specificity and clinical utility of this finding, since increased signal can also be seen with motion and other artifacts. ${ }^{3,4,9}$ Based on the findings in this study, we feel the superimposed increased signal from artifacts is such that it is not always possible to reliably detect infarction prospectively, using current equipment.

Edema occurs as a consequence of myocardial injury, resulting in an increase in both $T 1$ and $T 2$ relaxation times. ${ }^{14,17}$ Since, with our scanner, infarction resulted in increased MR signal, it is the $T 2$ effect that is responsible for lesion contrast; even the first echo images obtained with TE $=28 \mathrm{msec}$ show $T 2$ influence. Hence, one would expect that images with increased $T 2$ weighting, that is longer TR and/or TE, would bc bcst for visualizing myocardial infarction. Our data indicates that indeed longer TR images are preferable, though at the cost of increased imaging time. However, our longer-TE second echo images were of only limited utility, because of both the diminished signal-to-noise of longer-TE images, and the presence of high-signal motion artifact.

The endocardial border could be reliably seen in most of these studies on both the long and short TR images, supporting a role for MRI in assessing regional and global cardiac wall motion and function. ${ }^{1,6,10,11,15}$

We did not observe significant changes in the signal of injured myocardium compared to normal myocardium with reperfusion, a finding similar to one report by previous investigators, ${ }^{7}$ though this group did find significant differences subsequently. ${ }^{8}$ Since increased edema occurs following reperfusion, it is certainly possible that with either a different experimental protocol, or improvements in image quality, such changes would be demonstrable. ${ }^{8,13,16}$ Increases in both $T 1$ and $T 2$ relaxation times have been well described following myocardial injury, and our postsacrifice data provides further confirmation. However, statistically significant changes were not seen in vivo, in part because of image degradation from respiratory and cardiac motion. This is reflected in the large variation we found in our calculated relaxation times. Hence, quantitative manipulation of image data did not improve the efficacy of MRI.

In conclusion, we found that myocardial injury can be retrospectively identified on gated spin-echo images as regions of increased signal intensity. The endocardial border is also generally clearly demarcated. However, image quality is not good enough to permit accurate prospective identification of infarction, or to consistently show changes which occur immediately after reperfusion. MRI technology continues to undergo rapid improvement, and it is certainly possible that its efficacy will improve in the near future. 


\section{REFERENCES}

1. Buckwalter, K.A.; Aisen, A.M.; Dilworth, L.R.; Mancini, G.B.; Buda, A.J. Gated cardiac MRI: Ejectionfraction determination using the right anterior oblique view. AJR 147:33-37; 1986.

2. Buda, A.J.; Aisen, A.M.; Juni, J.E.; Gallagher, K.P.; Zotz, R.J. Detection and sizing of myocardial ischemia and infarction by nuclear magnetic resonance imaging in the canine heart. Am. Heart J. 110:1284-1290; 1985.

3. Filipchuk, N.G.; Peshock, R.M.; Malloy, C.R.; Corbett, J.R.; Rchr, R.B.; Buja, L.M.; Jansen, D.E.; Redish, G.R.; Gabliani, G.I.; Parkey, R.W.; Willerson, J.T. Detection and localization of recent myocardial infarction by magnetic resonance imaging. $\mathrm{Am}$. J. Cardiol. $58: 214-219 ; 1986$.

4. Fisher, M. R.; McNamara, M.T.; Higgins, C.B. Acute myocardial infarction: MR evaluation in 29 patients. AJR 148:247-251; 1987.

5. Higgins, C.B.; Herfkens, R.; Lipton, M.J.; Sievers, R.; Sheldon, P.; Kaufman, L.; Crooks, L.E. Nuclear magnetic resonance imaging of acute myocardial infarction in dogs: Alterations in magnetic relaxation times. $\mathrm{Am}$. J. Cardiol. 52:184-188; 1983.

6. Higgins, C.B.; Lanzer, P.; Stark, D.; Botvinick, F.; Schiller, N.B.; Lipton, M.J.; Crooks, L.E.; Kaufman, L. Assessment of cardiac anatomy using nuclear magnetic resonance imaging. $J$. Am. Coll. Cardiol. 5:775$815 ; 1985$.

7. Johnston, D.L.; Brady, T.J.; Ratner, A.V.; Rosen, B.R.; Newell, J.B.; Pohost, G.M.; Okada, R.D. Assessment of myocardial ischemia with proton magnetic resonance: Effects of a three hour coronary occlusion with and without reperfusion. Circulation 71:595-601; 1985.

8. Johnson, D.L.; Liu, P.; Rosen, B.R.; Levine, R.A.; Beaulieu, P.A.; Brady, T.J.; Okada, R.D. In vivo detection of reperfused myocardium by nuclear magnetic resonance imaging. JACC 9:127-135; 1987.

9. Johnston, D.L.; Thompson, R.C.; Liu, P.; Dinsmore, R.E.; Wismer, G.L.; Saini, S.; Kaul, S.; Rosen, B.R.; Brady, T.J.; Okada, R.D. Magnetic resonance imaging during acute myocardial infarction. Am. J. Cardiol. 57:1059-1065; 1986.

10. Kaul, S.; Wismer, G.L.; Brady, T.J.; Johnston, D.L.; Weyman, A.E.; Okada, R.D.; Dinsmore, R.E. Measurement of normal left heart dimensions using optimally oriented MR images. AJR 146:75-79; 1986.

11. McNamara, M.T.; Higgins, C.B.; Schechtmann, N.; Botvinick, E.; Lipton, M.J.; Chatterjee, K.; Amparo, E.G. Detection and characterization of acute myocardial infarction in man with use of gated magnetic resonance. Circulation 71:717-724; 1985.

12. Pflugfelder, P.W.; Wisenberg, G.; Prato, F.S.; Carroll, S.E.; Turner, K.L. Early detection of canine myocardial infarction by magnetic resonance imaging in vivo. Circulation 71:587-594; 1985.

13. Ratner, A.V.; Okada, R.D.; Newell, J.B.; Pohost, G.M. The relationship between proton nuclear magnetic resonance relaxation parameters and myocardial perfusion with acute coronary arterial occlusion and reperfusion. Circulation 71:823-828; 1985.

14. Rokey, R.; Verani, M.S.; Bolli, R.; Kuo, L.C.; Ford, J.J.; Wendt, R.E.; Schneiders, N.J.; Bryan, R.N.; Roberts, R. Myocardial infarct size quantification by MR imaging early after coronary artery occlusion in dogs. Radiology 158:771-774; 1986.

15. Stratemeier, E.J.; Thompson, R.; Brady, T.J.; Miller, S.W.; Saini, S.; Wismer, G.L.; Okada, R.D.; Dinsmore, R.E. Ejection fraction determination by MR imaging: Comparison with left ventricular angiography. Radiology 158:775-777; 1986.

16. Tscholakoff, D.; Higgins, C.B.; Sechtem, U.; Caputo, G.; Derugin, N. MRI of reperfused myocardial infarct in dogs. AJR 146:925-930; 1986.

17. Williams, E.S., Kaplan, J.L.; Thatcher, F.; Zimmerman, G.; Knoebel, S.B. Prolongation of proton spin lattice relaxation times in regionally ischemic tissue from dog hearts. $J$. Nucl. Med. 21:449-453; 1980.

18. Wesbey, G.; Higgins, C.B.; Lanzer, P.; Botvinick, E.; Lipton, M.J. Imaging and characterization of acute myocardial infarction in vivo by gated nuclear magnetic resonance. Circulation 69:125-130; 1984. 